\title{
LEVANTAMENTO ICTIOLÓGICO DE UM TRECHO DOS RIOS JACUI E JACUIZINHO NA ÁREA DE ABRANGENCIA DA FUTURA BARRAGEM DE DONA FRANCISCA
}

Ilca Marion Knewitz Bossemeyer, Maria Lacy Cezimbra Weis e Maria de Lourdes Souza Bier

Departamento de Biologia. Centro de Ciências Naturais e Exatas. UFSM. Santa Maria, RS.

RESUMO

o levantamento da fauna ictiolōgica num trecho dos rios Ja cuĩ e Jacuizinho, R.S., permitiu a identificação de vinte espēcies, sendo que os lambaris (Astyanax bimaculatus e. Astyanax fasciatus) e os cascudos (Hemiancistrus sp) foram os peixes mais abundantes e de distribuição uniforme nos meses de coleta, enquanto que o mussum (Symbranchus marmoratus) e o tambicu (oligosarchus hepsetus) foram os de ocorréncia mais rara.

SUMMARY

BOSSEMEYER, I.M.K.; WEIS, M.L.C. and BIER, M.L.S., 1981. Ictiologic Survery of Part of The Jacuí and Jacuizinho Rivers In The Area of The Future Dona Francisca Dam. Ciência e Natura (3) : 59-64.

The ictiologic survery of the fauna of part of the Jacuí and Jacuizinho rivers (RS - Brazil) lead to the identification of twenty species, Astyanax bimaculatus and Astyanax fasciatus as we 11 as Hemiancistrus $s p$ being more abundant and uniformly distributed during the months of collet while symbranchus marmoratus and oligosarchus hepsetus were the more seldom found fishes.

INTRODUÇÃO

Este levantamento foi realizado com a finalidade de iden tificar as espécies de peixes encontradas em um trecho dos rios Jacuí e Jacuizinho (RS - Brasil), na àrea de abrangência onde será construí da a barragem de Dona Francisca.

MATERIAL E METODOS

A ārea em estudo abrange o trecho do Rio Jacuí compreendi do entre o ponto $1\left(53^{0} 17^{\prime} 20^{\prime \prime}\right.$ de longitude oeste e $29^{\circ} 26^{\prime} 10^{\prime \prime}$ de la titude sul) e o ponto $6\left(53^{\circ} 14^{\prime} 40^{\prime \prime}\right.$ de longitude oeste e $29^{\circ} 17^{\prime} 8^{\prime \prime}$ de latitude sul) e o trecho do Rio Jacuizinho entre sua barra com o Ja

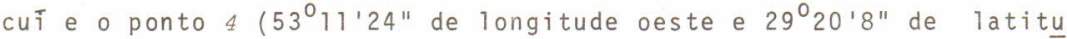
de sul). (Figura 1)

As coletas foram realizadas nos meses de fevereiro,março, 


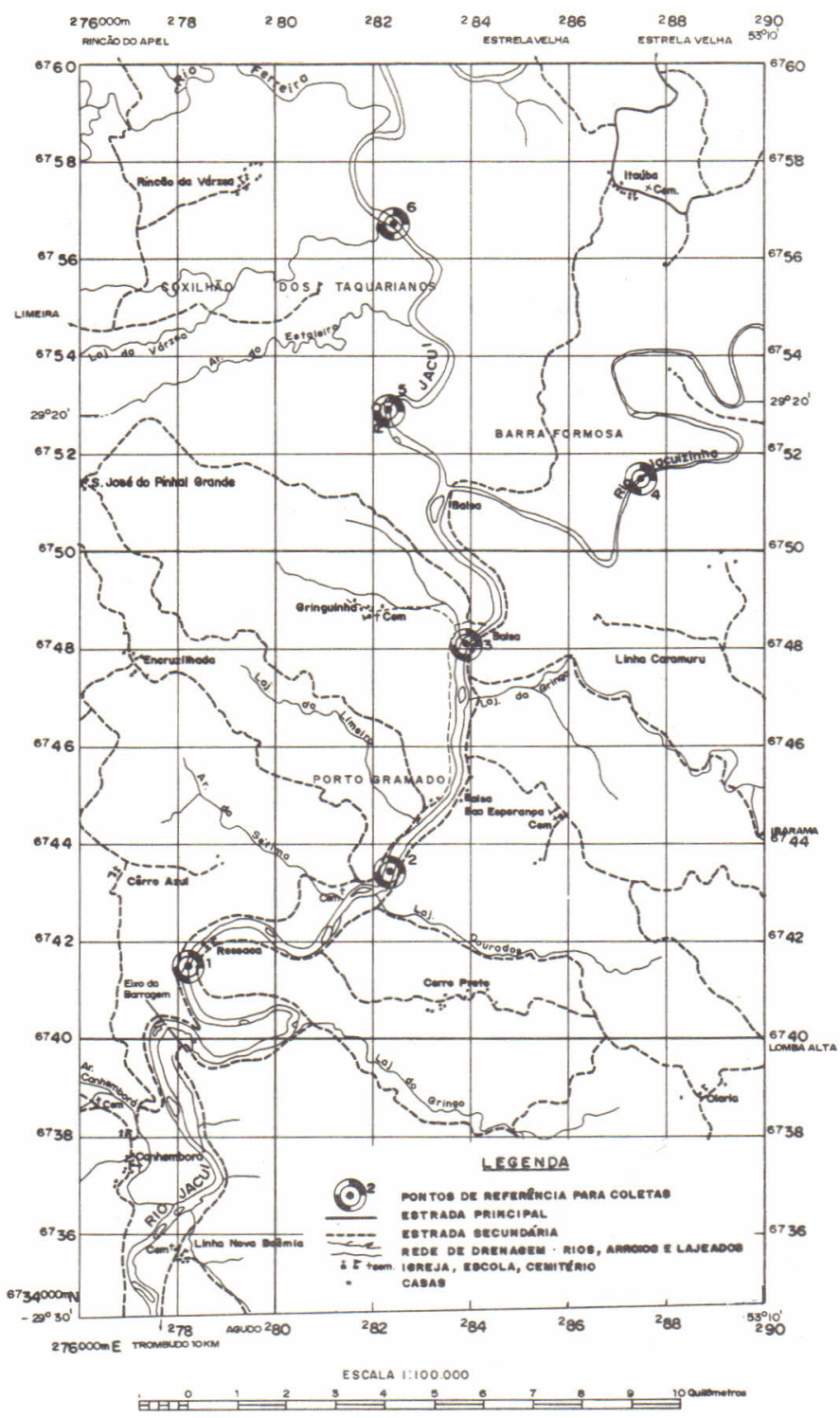

Figura 1. Mapa de localização dos pontos de coleta dos peixes ao lon go dos rios jacuí e Jacuizinho. 
abril, maio e junho de 1981, nos pontos 1, 2, 3, 4, 5 e 6. (Figura 1).

A captura dos exemplares da população em estudo deu-se de acordo com o disposto na Tabela I; de posse do número de espécies calculou-se os percen tuais de ocorrência mensal e total durante o período considerado (Tabela II).

No laboratōrio do Setor de Zoologia do Departamento de Bio logia da UFSM, os exemplares foram numerados, fixados em formol a $10 \%$ e conservados em ālcool a $70 \%$. Apōs sua identificação foram catalo gados e incluidos na coleção ictiolōgica do referido setor.

TABELA I. SUMARIO DAS COLETAS, INCLUINDO DATA, NOMERO DE INDIVIDUOS E ESPECIES, DOS PEIXES.

\begin{tabular}{lcc}
\hline DEATA & NO DE INDIVIDUOS & NO \\
\hline I981 & & \\
FEVEREIRO & 119 & 10 \\
MARÇO & 69 & 11 \\
ABRIL & 76 & 11 \\
MAIO & 78 & 10 \\
JUNHO & 140 & 13 \\
\hline
\end{tabular}

\section{RESULTADOS E COMENTARIOS}

Os 482 exemplares coletados (Tabela I) foram identificados com base na literatura consultada $(1,2,3,4,5,6,7$ e 8$)$ como per tencentes às seguintes categorias sistemáticas:

1) ORDEM: Cypriniformes

SUB-ORDEM: Characoidei

FAMILIA: Characidae

SUB-FAMILIA: Acestrorhynchinae

origosarcus jenynsii

oligosarcus hepsetus

2) ORDEM: Cypriniformes

SUB-ORDEM: Characoidei

FAMILIA: Characidae

SUB-FAMILIA: Tetragonopterinae

Astyanax bimacuzatus

Astyanax fasciatus

3) ORDEM: Cypriniformes

SUB-ORDEM: Characoide $i$

FAMILIA: Anostomidae

$$
\text { Leporinus obtusidens }
$$

4) ORDEM: Cypriniformes 
TABELA II. \% MENSAL E TOTAL DAS ESPECIES DE PEIXES IDENTIFICADOS NUM TRECHO DO RIO JACUI E JACUIZINHO,NO LOCAL DA FUTURA BARRAGEM DONA FRANCISCA.

\begin{tabular}{|c|c|c|c|c|c|c|c|c|c|c|c|c|c|}
\hline & \multicolumn{12}{|c|}{ MESẼS } \\
\hline & & \multicolumn{2}{|c|}{$\begin{array}{l}\text { FEVEREIRO } \\
\text { NọEX. } \%\end{array}$} & \multicolumn{2}{|c|}{$\begin{array}{l}\text { MARÇO } \\
\text { Nọ EX. }\end{array}$} & $\begin{array}{l}\text { ABRIL } \\
\text { Nọ EX. }\end{array}$ & & $\begin{array}{l}\text { MAII } \\
\text { EX. }\end{array}$ & $\%$ & \multicolumn{2}{|c|}{$\begin{array}{l}\text { JUNHO } \\
\text { Nọ EX. \% }\end{array}$} & \multicolumn{2}{|c|}{$\begin{array}{l}\text { TOTAL } \\
\text { No EX. \% }\end{array}$} \\
\hline oligosareus jenynsii & (tambicū) & 3 & 2,52 & 2 & 2,90 & - & - & - & - & 8 & 5,71 & 13 & 2,70 \\
\hline oligosareus hepsetus & (tambicū) & 1 & 0,84 & - & - & - & - & - & - & - & - & 1 & 0,20 \\
\hline Astyanax bimaculatus & (1 ambari.). & 28 & 23,53 & 16 . & 23,19 & 26 & 34,21 & - & - & - & - & 70 & 14,52 \\
\hline Astyanax fasciatus & (1 ambarî) & 32 & 26,89 & 15 & 21,74 & 17 & 22,36 & - & - & 15 & 10,71 & 79 & 16,39 \\
\hline Leporinus obtusidens & (piava) & 2 & 1,68 & 2 & 2,90 & 1 & 1,32 & - & - & - & - & 5 & 1,04 \\
\hline Schizodon fasciatus & (voga) & 7 & 5,89 & 7 & 10,14 & 4 & 5,26 & 1 & 1,28 & 2 & 1,43 & 21 & 4,36 \\
\hline Prochilodus scrofa & (grumatã) & - & - & - & - & 1 & 1,32 & 5 & 6,41 & - & - & 6 & 1,25 \\
\hline Curimatus sp & (biru) & - & - & - & - & - & - & - & - & 7 & 5,0 & 7 & 1,45 \\
\hline Hoplias malabaricus & (traîra) & - & - & 2 & 2,90 & - & - & - & - & 5 & 3,57 & 7 & 1,45 \\
\hline odontesthes sp & (peixe-rei) & 1 & 0,84 & 1 & 1,45 & - & - & - & - & 4 & 2,86 & 6 & 1,25 \\
\hline Loricariichthys sp & (cascudo) & 9 & 7,56 & - & - & - & - & - & - & - & - & 9 & 1,87 \\
\hline Rineloricaria sp & (cascudo) & - & - & 1 & 1,45 & - & - & 15 & 19,24 & 10 & 7,15 & 26 & 5,39 \\
\hline Plecostomus commerson & $2 i i(\operatorname{cascudo})$ & 4 & 3,36 & 4 & 5,80 & 1 & 1,32 & 1 & 1,28 & 4 & 2,86 & 14 & 2,90 \\
\hline Plecostomus wuchereri & (cascudo) & - & - & - & - & - & - & 1 & 1,28 & 4 & 2,86 & 5 & 1,04 \\
\hline Hemiancistrus $s p$ & (cascudo) & 32 & 26,89 & 13 & 18,84 & 18 & 23,68 & 50 & 64,10 & 75 & 53,57 & 188 & 39,0 \\
\hline Rhamdia sapo & (jundiā) & - & - & - & - & 2 & 2,63 & 2 & 2,57 & 1 & 0,71 & 5 & 1,04 \\
\hline Pimelodus, maculatus & (pintado) & - & - & 1 & 1,45 & 1 & 1,32 & 1 & 1,28 & 4 & 2,86 & 7 & 1,45 \\
\hline Crenicichla lacustris & (joana) & - & - & - & - & 4 & 5,26 & 1 & 1,28 & 1 & 0,71 & 6 & 1,25 \\
\hline Geophagus brasiliensi & is (acarā) & - & - & 5 & 7,25 & 1 & 1,32 & - & - & - & - & 6 & 1,25 \\
\hline Symbranchus marmoratu & us (mussum) & - & - & - & - & - & - & 1 & 1,28 & - & - & 1 & 0,20 \\
\hline TOTAL & & 119 & & 69 & & 76 & & 78 & & 140 & & 482 & \\
\hline
\end{tabular}


SUB-ORDEM: Characoidei

FAMILIA: Prochilodontidae

Prochilodus serofa

5) ORDEM: Cypriniformes

SUB-ORDEM: Characoidei

FAMILIA: Curimatidae

Curimatus sp

6) ORDEM: Cypriniformes

SUB-ORDEM: Characoide $i$

FAMILIA: Erythrinidae

Hoplias malabaricus

7) ORDEM: Mugiliformes

SUB-ORDEM: Mugildide $i$

FAMILIA: Atherinidae

odontesthes $s p$

8) ORDEM: Siluriformes

FAMILIA: Loricariidae

SUB-FAMILIA: Loricariinae

Loricariichthys $s p$

Rineloricaria sp

Plecostominae

Plecostomus commersoni $i$

$P$. wuchereri

Hemiancistrus $s p$

9) ORDEM: Siluriformes

FAMILIA: Pimelodidae

SUB-FAMILIA: Pimelodinae

Rhamdia sapo

Pimelodus maculatus

10) ORDEM: Perciformes

FAMILIA: Cichlidae

Crenicichla lacustris

Geophagus brasiliensis

11) ORDEM: Symbranchiformes

FAMILIA: Symbranchidae

symbranchus marmoratus

Os especimens coletados pertencem a vinte espëcies e a cin co ordens diferentes. Dentre as vinte espēcies, as mais comuns foram os lambarís (Astyanax bimaculatus e Astyanax fasciatus) e os cascu dos (Hemiancistrus $s p$ ) e as mais raras foram o mussum (Symbranchus marmoratus) e o tambicū (Olïgosarchus hepsetuis) (Tabela II).

Dentre as ordens, as que apresentaram maior variedade de espécies foram a dos Cypriniformes, seguida dos Siluriformes. Nas ordens Mugiliformes e symbranchiformes ocorreu apenas uma espécie em em cada uma. 
CONCLUSÕES

Dentre as vinte espēcies encontradas, os lambarís (Astyonax bimaculatus e Astyanax fasciatus) e os cascudos (Hemiancistrus sp)fo ram os peixes mais abundantes e de distribuição uniforme entre os espécimens identificados, enquanto o mussum (Symbranchus marmoratus) e o tambicú (Ozigosarchus hepsetus) foram os de ocorrência mais rara.

\section{AGRADE CIMENTO}

Os autores agradecem à Companhia Estadual de Energia Elé trica as condições e os meios oferecidos à coleta do material.

\section{REFERENCIAS BIBLIOGRAFFICAS}

1. BRITSKI, H. Peixes de água doce do Estado de São Paulo - Sistemá tica. IN: COMISSAO INTERESTADUAL DA BACIA PARANA-URUGUAI. PO Zuigão e Piscicultura. São Paulo, Faculdade de Saúde Püblica da USP e Inst. de Pesca, CPRN, S.A., 1970, 79-108 p.

2. Devincenzi, C.J. \& teague, G.W. Ictiofauna del Rio Uruguay Médio. Anales dez Museo de Hist. Nat., Montevideo, 5(4):1-109, 1942.

3. EIGENMANN, C.H. \& BRAY, W.L. A revision of the american Cichlidae. Anals N.Y. Acad. Sci., VII: 607-24, 1894.

4. FOWLER, H.W. Os peixes de água doce do Brasil. Arq. de Zoologia do Est. de S. Paulo, São Paulo, IX: 1-400, 1954.

5. GERY, J. Characoids of the Word, USA, TFH Publications. Inc. Ltd., 1977. 632p.

6. RIBEIRO, A. de M. "Fauna Brasiliense" - Peixes (Eleutherobranchios Aspiraphoros) - Physoclisti. Arq. Mus. Nac., Rio de Janeiro, 17: 1-827, 1915.

7. RINGUELET, R.A.; ARAMBURU, R.H. \& ARAMBURU, A.A. Los peces argen tinos de água doce. 1 ed. La Plata, Librart, 1967, 602 p.

8. WEBB, B.F. Fish Populations of the Avon-Heathcote Estuary. N.z. Journal of Marine and Freshwater Research, 6(4):570-601, 1 a bril, 1968.

Recebido em dezembro, 1981; aceito em dezembro, 1981. 